\title{
A COUNTABLE MINIMAL URYSOHN SPACE IS COMPACT
}

R. M. STEPHENSON, JR.

1. Introduction. A topological space $X$ is said to be a Urysohn space provided that every pair of distinct points of $X$ have disjoint closed neighborhoods. In [3] C. T. Scarborough and A. H. Stone prove that a countable minimal regular space is compact, and in [4] Scarborough asks if there exist noncompact minimal Urysohn spaces which are countable.

The principal result of this note is the following:

Theorem. A countable minimal Urysohn space is compact.

The terminology used here coincides with that in [3] and [4]. We shall denote the set of positive integers by $N$.

2. The theorem. According to [4, Theorem 14], a countable $U$ closed space has an isolated point. Our first lemma strengthens this result.

Lemma 1. Let $X$ be a $U$-closed space, let $I$ be its set of isolated points, and suppose that $X-I$ is countable. Then $\bar{I}=X$.

Proof. Suppose that there exists a nonempty open set $V$ such that $V \cap I=\varnothing$. Let $X-I=\left\{x_{n} \mid n \in N\right\}$. An inductive argument shows that there exists a descending sequence $V_{n}, n \in N$, of nonempty open subsets of $V$ such that for every $k \in N$ there exists a neighborhood $W_{k}$ of $x_{k}$ whose closure misses the closure of $V_{k}$. Then the filter base $\left\{V_{n} \mid n \in N\right\}$ is a $U$-filter on $X$ which has void adherence.

Lemma 2. Let $X$ be a $U$-closed space, $I$ its set of isolated points, and $\mathcal{F}$ a countable filter base on $I$. Then $\mathcal{F}$ has an adherent point.

Proof. Let $\mathcal{F}=\left\{F_{n} \mid n \in N\right\}$. For each $n$ choose a point $x_{n} \in \bigcap\left\{F_{i} \mid i \leqq n\right\}$ and let $C=\left\{x_{n} \mid n \in N\right\}$. If there is a point $x \in \bar{C}-C$, then $x$ is an adherent point of $\mathcal{F}$. If $\bar{C}=C$ and $\cap \mathcal{F}=\varnothing$, then the open- and-closed sets $\left\{x_{j} \mid j \geqq n\right\}, n \in N$, generate a $U$-filter on $X$ which has void adherence.

Proof of the Theorem. Let $X$ be a countable minimal Urysohn space.

In [4] Scarborough proves that a minimal Urysohn space is semiregular. In [2] Katětov proves that a semiregular absolutely closed space is minimal Hausdorff and that a Urysohn minimal Hausdorff

Received by the editors September 20, 1968. 
space is compact (this latter result is also obtained in [1]). Thus it suffices for us to prove that $X$ is absolutely closed.

Since $X$ is a Lindelöf space, an easy argument shows that if every countable open filter base on $X$ has an adherent point, then $X$ is absolutely closed.

Let $\mathcal{F}$ be a countable open filter base on $X$, and let $I$ be the set of isolated points of $X$. Then $\mathcal{F} \mid I$ is a filter base by Lemma 1 , so $\mathcal{F} \mid I$ and, hence, $\mathcal{F}$ have nonvoid adherence by Lemma 2 .

Corollary. A countable U-closed space is absolutely closed.

\section{REFERENCES}

1. N. Bourbaki, Espaces minimaux et espaces complètement séparés, C. R. Acad. Sci. Paris 212 (1941), 215-218.

2. M. Katětov, Über H-abgeschlossene und bikompakte Räume, Časopis Pěst. Mat. 69 (1940), 36-49.

3. C. T. Scarborough and A. H. Stone, Products of nearly compact spaces, Trans. Amer. Math. Soc. 124 (1966), 131-147.

4. C. T. Scarborough, Minimal Urysohn spaces, Pacific J. Math. 27 (1968), 611-617.

University of North Carolina at Chapel Hill 